\title{
How to Perform Phase II Trials in Psychiatry
}

\author{
Michel Bourin* \\ Neurobiology of anxiety and mood disorders, University of Nantes, France
}

Received: March 03, 2018; Accepted: April 30, 2018; Published: May 08, 2018

*Corresponding author: Michel Bourin, Neurobiology of anxiety and mood disorders, University of Nantes, France, Email: Michel.bourin@univnantes.fr

\begin{abstract}
Phase II clinical trials is a cornerstone of the drug development process since beyond the dose-effect study, phase II trials also largely participate to the evaluation of the drug efficacy. Due to the development of randomized, double-blind, placebo-controlled studies, phase II trials are actually much closed to phase III studies in terms of methodology.

Indeed, groups of patients should be uniform and representative with regard to the disease, baseline characteristics and expected response to the treatment. In the field of psychopharmacology as in many others, an important attention is also given to the evaluation of the clinical effect as well as to the sources of variability.
\end{abstract}

Keywords: Phase II; Clinicaltrial; Methodology; Psychopharmacology; Psychotropic drugs

\section{Introduction}

The purpose of the phase II trial is to detect pharmacodynamics activity in the patientand determinethe range of active doses. Phase III trials subsequently indicate whether the pharmacodynamics activity is also therapeutic. Phase II is particularly delicate since a range of truly active doses must be used to confirm whether the drug is clinically efficient or not. On the basis of the findings of the phase I trial, tolerated doses are studied in the healthy volunteer, generally over the broadest range possible, which may require the participation of a considerable number of patients. Phase II trials are generally conducted in parallel groups in which three doses of the drug (D1, D2 and D3) are studied versus a placebo and a control product. In any event, it is absolutely necessary to use a placebo in phase II and to provide irrefutable proof that the drug is more effective than the placebo $[1,2]$. In the past, phase II trials were often open, non-controlled studies involving few patients. This practice proved unsuitable since the new drug seemed effective in the absence of comparison with a placebo or control product. Except for special cases, the customary practice now is to perform controlled, randomized studies in parallel groups in phase II and later trials [3]

\section{Phase II methodology}

The methodology of phase II trials differs only slightly from that of phase III. It requires the use of selected patients, who are hospitalized when studying antipsychotics in schizophrenic patients. For other pathologies such as sleep disorders, anxiety, depression it is possible to work with non-hospitalized patients. The groups studied must be homogeneous as to age, sex and the clinical form of the disease.

The main purpose of studying the relation between drug dose and effect in man is to define the limits of effective doses in relatively small groups of patients. A lower limit or threshold of clinical effectiveness is determined, which is the minimal dose producing a significant effect. An upper limit or toxicity threshold is the maximal dose that can be administered without producing significant toxicity. The dose-effect study thus includes an analysis of changes in effects as a function of the dose administered and possibly of the drug concentrations in an easily sampled biological medium (most often plasma but also urine or saliva).

The study of the dose-effect relation also suggests which administration route will provide the expected effect, detects the possible presence of active metabolites of the drug, reveals any phenomena of tolerance or sensitization of receptor sites and allows the study of possible interactions among various drugs.

\section{Factors of variation in the dose-effect relation}

Although the main purpose of the study of drug-effect relations seems simple in a given patient, the situation is much more complex when the relations are defined in a population of patients. The purpose of clinical trials of new drugs conducted with relatively small groups of patients is to determine a general law applicable to the vast majority of patients who will ultimately receive the drug. In fact, there is considerable difficulty in applying this general law to individual treatment since the doseeffect relation is subject to the influence of many factors:

1.The characteristics of patients: age, sex, weight, body surface area.

2.The pathologic state: etiology and course of the disease, associated pathology.

3. Inter individual variations in drug pharmacokinetics [4].

4. The presence of one or more active metabolites whose kinetics can differ from that of the parent product; variation of effects depending on environmental factors, schedules, food, living conditions, stress or endogenous factors (affinity or number of cell receptors). 
5. The possible presence of pharmacokinetic and/or pharmacodynamic drug interactions.

All of these factors affect the individual relation between the dose administered and the effect observed, which makes the dose-effect relation during phase II and III clinical trials quite "fragile" and finally purely indicative. Any subsequent medical treatment will require an individual adaptation of the dosage.

\section{Determination of the clinical effect}

Determination of the clinical effect of a drug requires, first of all, that the variation of a drug-related parameter be distinguished from variations related to a spontaneous change in the state of the patient or to an error in measuring the parameter. On the one hand, this implies that the spontaneous variability of the parameter is known, either by measurement of the parameter both before and after drug administration or in the same experimental conditions when patients receive a placebo. On the other hand, determination of the clinical effect implies that the sensitivity and reliability of the method used to ascertain the effect are known, which ensures that the error in measurement is slight relative to variations of the drug-related parameter [5]

Subsequently, the method of measuring the clinical effect depends on the type of effect observed. Schematically, two situations are possible:

\section{Effect analyzed according to a binary mode}

In this situation, the effect is either present or absent. This is true, for example, of the therapeutic effects of antiepileptic drugs (presence or absence of seizure). The effect can also correspond to a significance threshold dependent on spontaneous variability and the pathologic situation. The dose-effect curve shows the proportion of subjects responding to treatment for each of the doses studied. When a therapeutic effect is studied, it is maximal when all of the patients are treated efficaciously. The dose-effect relation is most often of sigmoid form.

\section{Effects analyzed according to a continuous mode}

When the effect shows continuous variation from 0 (absence of effect) to a maximum during an increase of doses, the concentration-effect relation is defined by a parametric model. In practice, it is rarely possible in man to measure a maximal effect, and the effect is not always proportional to the blood concentration of the drug. The dose-effect relation then most often assumes a sigmoid form whose middle part corresponds to a linear relation between effect and dose. More complex models have been proposed for the analysis of concentrationeffect relations, either by using logarithmic transformation of the concentration (corresponding to a linear approximation of the hyperbolic model in the zone of effects representing 20 to $80 \%$ of the maximal effect) or by integrating the pharmacokinetic and pharmacodynamics model by calculating the concentrations of the drug at the "theoretical site of the effect."

\section{Method of studying the dose-effects relationship}

Studies of the relation between the dose administered and the effect measured, as well as the search for a range of effective and well-tolerated doses, are performed during phase II clinical trials. These initial trials in patients come after phase I studies in healthy volunteers during which the maximal limit of drug doses administrable to humans and the pharmacokinetics of the drug have been determined.

\section{Administration of single doses}

During administration of a single dose to a group of patients, changes in the effect and plasma concentrations of the drug are studied over a period of time. If the effect is measured in binary mode, the proportion of patients who attain the efficacy threshold is determined as well as the time required to reach this threshold and maintain it. The plasma concentration corresponding to the efficacy threshold is also determined. In continuous mode, the appearance of the effect is noted as well as the maximum reached and the fall. The dose-effect relation can be studied by considering the maximal effect observed for each drug dose. The study of the plasma concentration-effect relation can be based on an analysis of the plasma concentration corresponding to the maximal effect or on correlations between the area under the curve for plasma concentration time and effect time.

Simultaneous measurement of the drug effect and plasma concentrations often reveals a hysteresis phenomenon when (as is quite frequently the case after intravenous administration) the effect persists during the fall in plasma concentrations. This phenomenon can be related to the persistence of the drug at the activity site, the late elimination of an active metabolite, or more rarely the sensitization of receptor sites during the study.

\section{Administration of repeated doses}

During repeated administrations of the drug, changes in effect are observed as plasma concentrations gradually increase until a plateau is reached. The effect is then measured in steady state after a period of administration equal to at least 5 times the elimination half-life of the drug.If the effect is measured in binary mode, the number of doses and plasma concentrations required to reach the efficacy threshold are noted. The proportion of "responders" attaining clinical efficacy is determined for the steady state of plasma concentrations corresponding to each dose. If the effect is measured in continuous mode, changes are studied in terms of the time-points during administration of repeated doses when plasma concentrations increase and then during the steady state of these concentrations.

\section{Experimental Protocols}

Two types of experimental protocols are possible, depending on whether the drug is administered in a single dose or repeated doses.

Protocol in parallel groups: The basal state of patients is determined during an initial study most often performed single-blind with a placebo. The patients are randomly divided into parallel groups and then, depending on the administration procedure, receive a single dose per group or repeated doses for a period sufficient to reach the steady state of plasma 
concentrations. Several groups of patients are thus composed (one per dose), while a control group receives a placebo in the same experimental conditions if the pathology is not too severe. When possible, a 5-arm controlled study is performed: three doses of the compound are compared with a control drug and a placebo $[6,7]$.

Crossover protocol: In this type of protocol, the patient is his own control. Basal state is determined for each patient, and the different doses of the drug are administered successively and studied for their effect. This protocol can be used for administration of the drug in a single dose, in which case each dose is followed by a sufficient wash-out period, so that all effects are dissipated before administration of the following dose. It can also be used for administration of repeated doses, in which case each dose level is of sufficient duration to allow the steady state of plasma concentrations to be reached. In these crossover protocols, the order of the doses administered to each subject can be determined (e.g. in increasing order) or randomized. Placebo periods can be included, either during the protocol when administration is randomized or most often at the end of the trial when doses are administered in increasing order. These placebo periods allow the stability of the pathologic state of patients to be verified and demonstrate the possible existence of a placebo effect related to administration of a therapeutic drug. In fact, this type of protocol is very difficult to carry out, except with healthy volunteers in phase I trials when some pharmacodynamic parameters are measured.

Practical problems: Regardless of the type of protocol chosen for determination of the range of effective tolerated doses during phase II clinical trials, certain practical problems are constantly encountered:

\section{Criteria of patient selection}

As the search for effective doses is carried out in small groups of patients, it is advisable that their homogeneity be as great as possible concerning age, pathology, course of the disease, etc. Intercurrent factors that could hinder interpretation of the measured clinical effect should be eliminated.

\section{Criteria of therapeutic efficacy}

The criteria for judging the effect in phase II trials may not be strictly therapeutic. However, if the measured effect is therapeutic in nature, the type of effect observed (binary or continuous variation mode) influences analysis of trial results. It is thus advisable to define the criteria of therapeutic efficacy initially and carefully. The same is true for the criteria of clinical and biological tolerance of the drug.

\section{Choice of doses}

The drug doses to be studied in patients range from the minimal dose, most often corresponding to that which has produced a measurable pharmacologic effect in healthy volunteers during phase I trials, to a maximal dose generally lower than that tolerated in phase I trials. The number of doses to be tested is variable, most often from 3 to 6 , and should remain within the limits of feasibility of the protocol.

\section{Number of subjects}

The number of patients receiving a drug dose (single or repeated) depends mainly on the variability of the parameter considered for measuring the expected effect (and its amplitude) [8].

\section{Definition of the minimum treatment period}

During administration of repeated doses of the drug, the effect should be measured after a steady state is reached for plasma concentrations. The treatment period for each dose level should thus be equal to 5 times the elimination half-life of the drug. This is not true when drug kinetics is not linear, the elimination half-life changes during treatment, or active metabolites are eliminated over a long period of time. In these cases, longer treatment periods are required, and measurement of plasma concentrations of the drug and/or its metabolites is may be required.

\section{Difficult phase II trials}

The classical phase II methodology described above is ideally applicable to situations in which an effect can be easily demonstrated in man. The determination of the optimal dose is much more difficult when the desired effect is obtained late (e.g. an antidepressant effect); the difficulties are major when the treatment is intended to be preventive in the long run (efficiency of the mood stabilizers)

\section{Conclusion}

The classical phase II methodology as described above is ideally applicable to situations where a clear drug-response could be demonstrated. During a long time, psychopharmacology had very poor phase II studies due to insufficiency in evaluation criteria. Nowadays, in many fields as depression or anxiety, it could be shown a dose-effect relationship with drugs as various as venlafaxine or SSRI $[9,10]$. Nevertheless, the determination of optimal dose could remain an important problem when there is no readily apparent therapeutic effect or predictive pharmacologic effect as in the treatment of schizophrenia. In this context, there is a need to improve clinical trials methodology [11] including the quality of scoring since rater in clinical trials could also contribute to the dose-effect variability [12]. In a near future, we could also postulate that the pharmacogenetic approach will help to optimize drug development and therapeutics, increasing efficacy and safety and reducing side-effects and unnecessary costs.

\section{References}

1. Lavori PW. Placebo control groups in randomized treatment trials: a statistician's perspective. Biol Psychiatry. 2000;47(8):717-723.

2. Young SN, Annable L, Stat D. The ethics of placebo in clinical psychopharmacology: the urgent need for consistent regulation. J Psychiatry Neurosci. 2002;27(5):319-321.

3. Perkins KA, Lerman C. An efficient early phase 2 procedure to screen medications for efficacy in smoking cessation. Psychopharmacology (Berl). 2014;231(1):1-11. doi: 10.1007/s00213-013-3364-6 
4. Ahmed AI, van den Elsen GA, Colbers A, Kramers C, Burger DM, et al. Safety, pharmacodynamics, and pharmacokinetics of multiple oral doses of delta-9-tetrahydrocannabinol in older persons with dementia.Psychopharmacology (Berl). 2015;232(14):2587-2595. doi: 10.1007/s00213-015-3889-y

5. Chaumet-Riffaud P, Wolmark Y, Pere JJ, Goulley F. Therapeutic trials in Alzheimer disease. Selection, recruitment and stratification. Therapie. 1993;48(3):201-205.

6. Keck PE, Welge JA, McElroy SL, Arnold LM, Strakowski SM. Placebo effect in randomized, controlled studies of acute bipolar mania and depression. Biol Psychiatry. 2000;47(8):748-755.

7. Walsh BT, Seidman SN, Sysko R, Gould M. Placebo response in studies of major depression: variable, substantial, and growing. JAMA. 2002;287(14):1840-1847.

8. Boutitie F, Bellissant E, Blanchard J, Boissel JP, Cauquil J, Chauvin F Derzko et al. Monitoring of clinical trials and interim analysis. 2 . Statistic methods. Therapie. 1992;47(4):351-355.
9. Baker CB, Tweedie R, Duval S, Woods SW. Evidence that SSRI dose response in treating major depression should be reassessed: a metaanalysis. Depress Anxiety. 2003;17(1):1-9.

10.Smith D, Dempster C, Glanville J, Freemantle N, Anderson I. Efficacy and tolerability of venlafaxine compared with selective serotonin reuptake inhibitors and other antidepressants: a meta-analysis. Br J Psychiatry. 2002;180:396-404.

11. Klein DF, Thase ME, Endicott J, Adler L, Glick I, Kalali A, et al. Improving Clinical Trials American Society of Clinical Psychopharmacology Recommendations. Arch Gen Psychiatry. 2002;59(3):272-278. doi:10.1001/archpsyc.59.3.272

12. Petkova E, Quitkin FM, McGrath PJ, Stewart JW, Klein DF. A method to quantify rater bias in antidepressant trials. Neuropsychopharmacology. 2000;22(6):559-565. 